\title{
REMOTE CONTROL OF A NETWORKED MOBILE ROBOT USING AN IMMERSIVE LOCOMOTION INTERFACE
}

\author{
Kazumasa Yamazawa, Masaki Tawada, and Naokazu Yokoya \\ Graduate School of Information Science \\ Nara Institute of Science and Technology \\ 8916-5 Takayama, Ikoma, Nara, Japan 630-0192 \\ E-mail: yamazawa@is.naist.jp
}

\begin{abstract}
Recently, the concept of behavioral media using a mobile robot has emerged in tele-robotics, where the mobile robot behaves as a user's second body or an agent in a remote site. An important issue in behavioral media is how to control the robot intuitively immersing a user into a remote site. This paper describes a new approach which combines locomotion interface and immersive projection display of a remote site for controlling a networked mobile robot. We have developed a prototype system and have evaluated the usability of the system with experiments.
\end{abstract}

\section{INTRODUCTION}

Behavioral media using a mobile robot is expected to have an important role in some applications such as rehabilitation, virtual tour, active surveillance, and tele-operation in extreme environments. One of the most important issues concerning behavioral media is how to control the robot intuitively immersing a user into a remote site.

A number of remote control and tele-operation techniques have been investigated for controlling a mobile robot in robotics. Typical devices for controlling the robot are foot pedals[1], joysticks[2,3,4], and a mouse[5,6]. Immersion into a remote site has been achieved by presenting omni-directional stereo views on a head-mounted display[7,8].

In this paper, we describe a new approach which combines locomotion interface and immersive projection display of a remote site for controlling a networked mobile robot. In our approach, a user controls a mobile robot in a remote site simply walking on a treadmill located in an immersive projection display environment. Locomotion interface based on walking behavior provides the user with intuitive control of the robot and immersive projection display of omni-directional live video does the user with the feeling of rich presence in the remote site. The rest of this paper describes a prototype system and its evaluation with experiments.

\section{PROTOTYPE SYSTEM}

\subsection{Overview of prototype system}

Fig. 1 shows the configuration of prototype system. A user walks on a treadmill to control a remote mobile robot. Walking information is received by a PC for treadmill control. The PC controls the treadmill and transports walking information to the mobile robot via network. In the mobile robot site, a PC for mobile robot moves the robot based on user's walking, and transports omni-directional video acquired by an omni-directional camera to the user's site via network. The transported omni-directional video is received by a $\mathrm{PC}$ for video reception, and is transported to a graphics WS via DV recorder. The omni-directional video is transformed to appropriate perspective video, and is projected onto an immersive display. The user can control the remote mobile robot by repeating the above process. The configuration of the prototype system is described in the following.

Mobile robot (Wheelchair of Misawa: M-Smart)

Misawa M-Smart is used as the base of mobile robot. MSmart is an electric wheelchair which is usually controlled with the joystick on the right armrest. The mobile robot has been rebuilt so that it can be controlled by digital input from a laptop PC. Fig. 2 (left) shows the appearance of the mobile robot. The mobile robot can move forward-andbackward and rotate right-and-left.

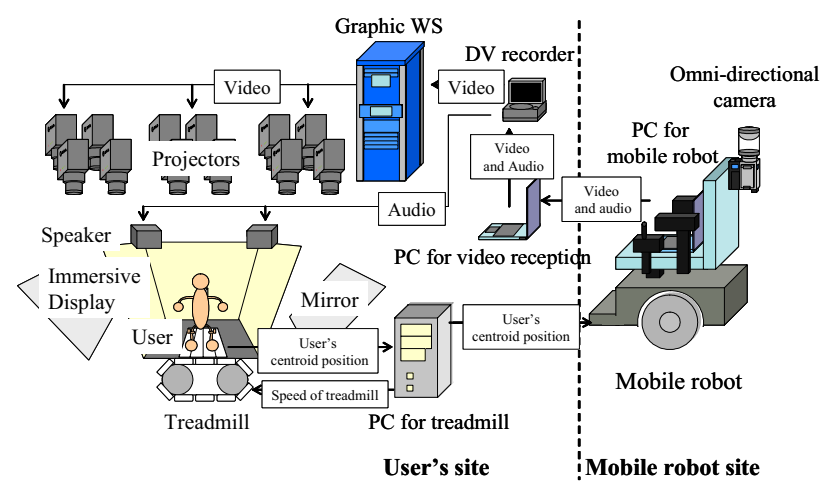

Fig. 1. Configuration of prototype system. 


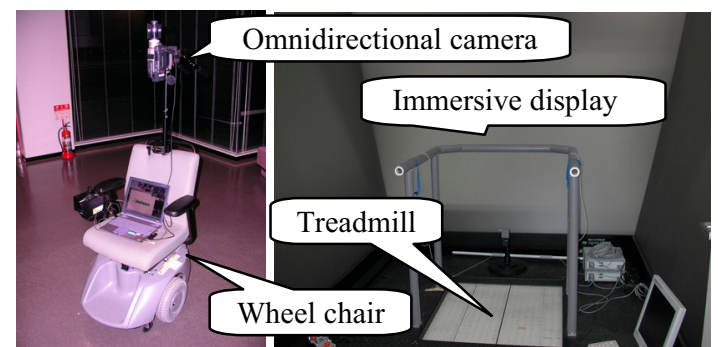

Fig. 2. Appearance of mobile robot (left), immersive display, and treadmill (right).

\section{Omni-directional camera (HyperOmni Vision)}

The omni-directional camera is mounted on the mobile robot so that it is located at almost the same height (about $160 \mathrm{~cm}$ ) as user's viewpoint. The omni-directional camera acquires a 360-degree view around the robot. The omnidirectional camera satisfies the single viewpoint constraint and thus the acquired omni-directional video can be transformed to common perspective video as well as panorama video suitable for presentation[9].

PC for mobile robot (SONY: PCG-R505FR-D)

It is carried on the mobile robot, and controls the robot based on user's walking information which is received via network. Simultaneously, it transports the acquired omnidirectional video and audio to the user's site via network.

Treadmill (SOLIDRAY: WalkMaster)

The belt of the treadmill can be moved into all directions on a 2D plane. Therefore, the user can walk in all directions on the $2 \mathrm{D}$ plane. The user wears the magnetic sensors on both knees. The belt is moved so that the centroid of both knees is kept at the center of the treadmill. The user can walk at the fixed point on the treadmill [10]. The position of the centroid is used not only for controlling the treadmill but also for controlling the mobile robot by transporting the position of the centroid via network.

\section{PC for treadmill}

It controls the treadmill based on information from the magnetic sensor. Simultaneously, it transports user's waking information to the mobile robot site.

Immersive display (SOLIDRAY: VisualValley)

It consists of three large slanted screens (see Fig. 2 (right)) and corresponding twelve projectors (four projectors per screen). It displays video transported from the mobile robot.

Graphics WS (SGI: ONYX3800)

It transforms omni-directional video to common perspective video for the immersive display.

\section{PC for video reception}

It receives the stream which includes video and audio via network and sends the stream to the DV recorder.

\section{DV recorder}

It receives the stream, sends the video to Graphics WS, and outputs the audio to the speakers.

\subsection{Network transmission}

The system has two network transmissions. One is videoand-audio stream transmission. The other is operation instruction transmission. In video-and-audio stream transmission, the mobile robot site is the sender and the user's site is the receiver. On the other hand, in operation instruction transmission, the user's site is the sender and the mobile robot site is the receiver.

For transporting omni-directional video, we use DV commXP software made by Fatware Co. DVcommXP is based on RTP Payload Format for DV Video (RFC 3189) [11]. DVcommXP can transport high-resolution DV data (720x480, 30fps) using 30Mbps bandwidth via network. In this study, we set the framerate to $10 \mathrm{fps}$ for bandwidth of wireless network. The mobile robot site sends the omnidirectional video from the omni-directional camera mounted on the mobile robot. The user's site receives the omnidirectional video and transfers the video to the graphic WS via the DV recorder. The graphic WS transforms omnidirectional video to common perspective video and projects it on the immersive display.

The user's walking action is estimated by using magnetic sensors worn on both knees. Walking information is then transported to the PC on the mobile robot by using TCP/IP. At the mobile robot site, the PC transforms the received walking action to adequate instructions for the mobile robot and controls the mobile robot. The mobile robot can move only forward-and-backward and can rotate right-and-left as mentioned earlier. Therefore, the user's forward-and-backward translation and right-and-left translation should be reflected to forward-and-backward translation and right-and-left rotation of the mobile robot.

\subsection{Video presentation}

The transformed video is presented on the immersive display. First, the graphic WS transforms the omnidirectional video transported from the mobile robot via network to twelve common perspective videos corresponding to the number of projectors in real-time using an image warping technique [12]. Next, each common perspective video is presented on the immersive display via the associated projector. Moreover, for presenting a backward scene of the mobile robot, its common perspective video is generated and is presented on the upper part of the front screen as a rearview (see Fig. 3(b)). 


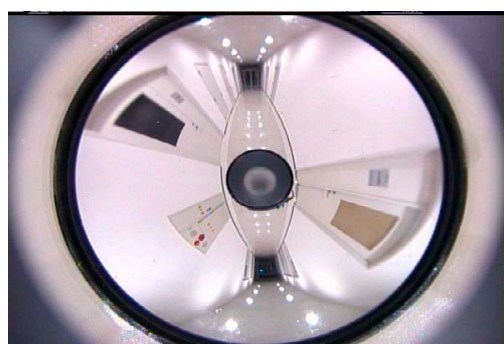

(a) Omni-directional image.

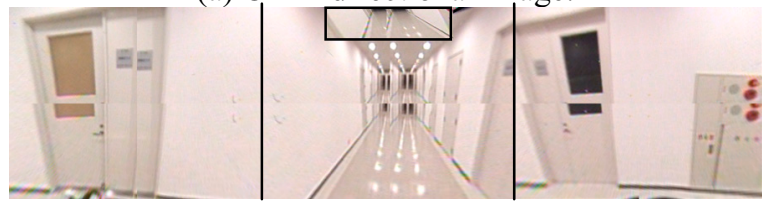

(b) Common perspective images for three screens.

Fig. 3. Omni-directional image and corresponding common perspective images projected on three screens.

\section{EXPERIMENTS}

\subsection{Overview of experiments}

We have experimented with the prototype system. The user's and mobile robot sites are located in different buildings. The distance between both sites is about $200 \mathrm{~m}$. The user wears magnetic sensors on both of knees, watches the video capturing the mobile robot site, and controls the mobile robot using the proposed locomotion interface. The network between both buildings is a wired 100Mbps connection. The network between the building and mobile robot is a wireless $54 \mathrm{Mbps}$ connection. We have set the framerate of video to 10 frames/sec. adjusting DVcommXP and have set the maximum speed of mobile robot to $2 \mathrm{~km} / \mathrm{h}$. Fig. 4 shows the appearance of experimental environments.

We have confirmed that the user can control the remote mobile robot by walking. The time delay from acquiring an omni-directional image to presenting it on the screen via network is about 1 second. The time delay from the beginning of user's walking to the beginning of the movement of mobile robot is also about 1 second. The delay is mainly caused by network delay. In the present implementation, the user must consider the delay of at most 2 seconds. Evaluation of the prototype system is described in the following section.

\subsection{Evaluation of usability}

\subsubsection{Tasks for evaluating usability}

For evaluating the usability, we have defined the following three tasks. Subjects know the size and moving mechanism of the remote robot before the tasks. Subjects control the mobile robot for tasks and some quantitative evaluation is carried out.

\section{Task 1: Stopping in front of an obstacle}

The mobile robot approaches an obstacle as close as possible and stops in no contact. The robot moves only straight forward. The distance between the starting point
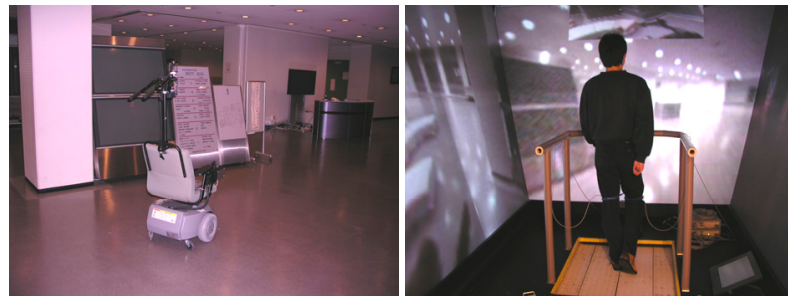

Fig. 4. Appearance of mobile robot site (left) and user's site (right) in experiment.

and the obstacle is $4.25 \mathrm{~m}$. The purpose of this task is to examine how much the delay affects the operation requiring the sense of distance.

\section{Task 2: Moving to a target point}

The mobile robot moves to a target point that the mobile robot cannot reach only by moving forward from the initial state of position and orientation. There is no obstacle in this task. When the task starts, the user can see the target point via the immersive display. The user can control the mobile robot within his/her discretion. The distance between the starting point and the target point is $5.60 \mathrm{~m}$. The user needs to move forward, rotating when the target point is not ahead of the mobile robot. The purpose of this task is to evaluate the intuitiveness of correspondence between user's movement and mobile robot movement.

Task 3: Avoiding obstacles and moving to a target point

The mobile robot avoids obstacles and moves to a target point. The distance in a straight line between the starting point and the target point is $6.70 \mathrm{~m}$. It is required to control the mobile robot without a collision with obstacles. The robot is required a rather long movement for avoiding obstacles. When the task starts, the user can see the target point via the immersive display. The purpose of this task is to evaluate the total maneuverability of the system.

The number of subjects for each task is nine. All of the subjects are beginners for using the system.

\subsubsection{Results and discussion}

Table 1 shows the results of task 1 including the operation time and the distance from the stopping point to the obstacle. The negative value means the distance between the obstacle and the stopping point after collision. The robot moves about $1 \mathrm{~m}$ in the total time delay of 2 seconds when it runs at $2 \mathrm{~km} / \mathrm{h}$. The results are within $1 \mathrm{~m}$. The subjects who are required much time can control the robot close to the obstacle. It should be noted that subjects are required to pay attention to the time delay when the distance between the robot and the obstacle is less than the distance to which the robot moves in the time delay.

Fig. 5 shows the results of task 2 including the operation time and the trajectory of the mobile robot. Few subjects changed the direction of the robot to the target point at first and moved straight forward. Many other subjects moved and rotated to the target point simultaneously. Since the distance between the starting point and the target point is large enough, the subjects could 
Table 1. Results of task1: Operation time and distance between stopping point and obstacle.

\begin{tabular}{|c|c|c|c|c|c|c|c|c|c|}
\hline Operator & a & b & c & d & e & f & g & h & i \\
\hline Operation time (sec.) & 14 & 16 & 12 & 22 & 17 & 12 & 13 & 22 & 15 \\
\hline Distance (cm) & 1 & -35 & 40 & -5 & 70 & 35 & 100 & -10 & 10 \\
\hline
\end{tabular}

adjust the direction of the robot to the target point repeatedly. It is confirmed that subjects can control the robot intuitively corresponding subjects' movement and the mobile robot movement when the subjects do not need to pay attention to obstacles.

Fig. 6 shows the results of task 3 including the operation time and the trajectory of the mobile robot. In this task, the skill of controlling the robot correctly for avoiding obstacles is required. Some subjects are required much time for the task. We observed that the reasons for this were either the speed is reduced for controlling the robot, or changing direction is repeated for avoiding the obstacles. Many subjects have reported that the task is difficult due to the followings: (i) there is no sense other than vision from the environment around the robot, (ii) the resolution of presented video is low, (iii) it is difficult to feel the distance

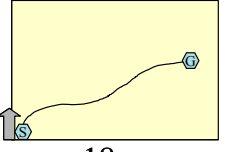

a: $18 \mathrm{sec}$

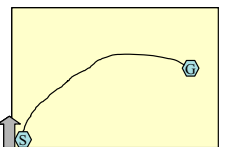

$\mathrm{d}: 19 \mathrm{sec}$

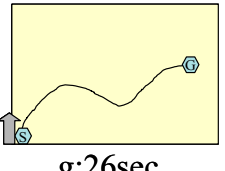

$\mathrm{g}: 26 \mathrm{sec}$
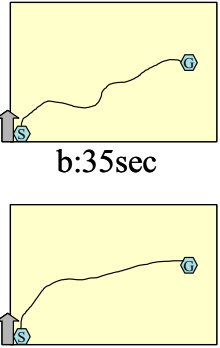

$\mathrm{e}: 22 \mathrm{sec}$

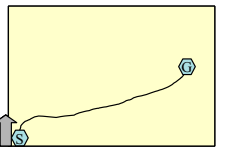

$\mathrm{h}: 26 \mathrm{sec}$
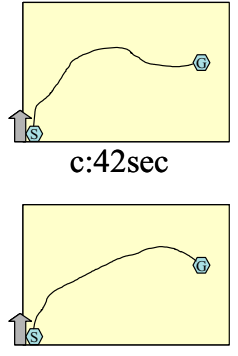

$\mathrm{f}: 21 \mathrm{sec}$

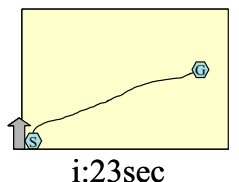

Fig. 5 Results of task 2: Operation time and trajectory.

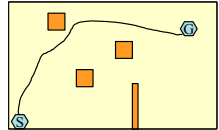

$\mathrm{a}: 41 \mathrm{sec}$

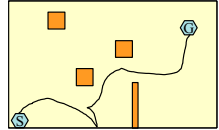

$\mathrm{d}: 70 \mathrm{sec}$

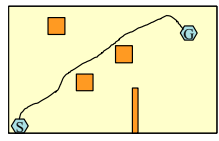

$\mathrm{g}: 50 \mathrm{sec}$

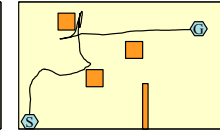

b:93sec

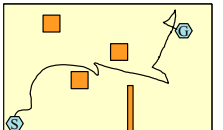

e: $135 \mathrm{sec}$

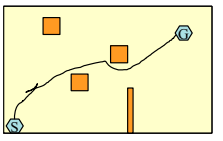

$\mathrm{h}: 50 \mathrm{sec}$

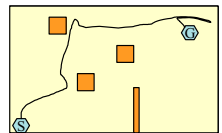

c: $91 \mathrm{sec}$

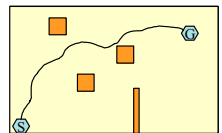

f: 132 sec

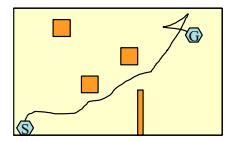

i:71sec
Fig. 6 Results of task 3: Operation time and trajectory. between the robot and obstacles, and (iv) the time delay is long.

\section{CONCLUSION}

We have constructed a remote control system for a mobile robot with a locomotion interface and an immersive display. The usability of the system has been evaluated with experiments.

Future work includes the followings. For the time delay, the graphics WS will show the estimated present location of the mobile robot on the immersive screen by drawing CG of the robot. The mobile robot should be equipped with obstacle detecting sensors. When the sensor detects a close obstacle, the mobile robot and the treadmill should stop for feedback to a user.

\section{REFERENCES}

[1] D. G. Caldwell, A. Wardle, and M. Goodwin, "Tele-presence: visual, audio and tactile feedback and control of a twin armed mobile robot," Proc. IEEE Int. Conf. on Robotics and Automation, Vol. 1, pp. 244-249, 1994.

[2] I. Elhajj, N. Xi, and Y. Liu, "Real-time control of internet based teleoperation with force reflection," Proc. Int. Conf. on IEEE Robotics and Automation, vol. 4, pp. 3284-3289, 2000.

[3] P. Jensfelt and S. Kristensen, "Active global localization for a mobile robot using multiple hypothesis tracking," IEEE Trans. on Robotics and Automation, vol. 17, pp. 748-760, 2001.

[4] D. A. Bell, J. Borenstein, S. P. Levine, Y. Koren, and J. Jaros, "An assistive navigation system for wheelchairs based upon mobile robot obstacle avoidance," Proc. IEEE Int. Conf. on Robotics and Automation, vol. 3, pp. 2018-2022, 1994.

[5] D. Schulz, W. Burgard, D. Fox, S. Thrun, and A.B. Creemers, "Web interfaces for mobile robots in public places," IEEE Robotics and Automation Magazine, Vol. 7, No. 1, pp. 48-56, 2000 [6] H. Hirukawa, T. Matsui, H. Onda, K. Takase, Y. Ishiwata, and K. Konaka, "Prototypes of teleoperation systems via a standard protocol with astandard human interface," Proc. IEEE Int. Conf. on Robotics and Automation, Vol. 2, pp. 1028-1033, 1997.

[7]. N. Yokoya, K. Yamazawa, and H. Takemura, "Interactive media-oriented applications of an omnidirectional video camera," Proc. World Automation Congress, Vol.13, pp.11-16, 2002.

[8]. R. Kurazume and S. Hirose, "Development of image stabilization system for remote operation of walking robots," Proc. IEEE Int. Conf. on Robotics and Automation, Vol.2, pp.1856-1861, 2000.

[9]. K. Yamazawa, Y. Yagi, and M. Yachida, "Omnidirectional imaging with hyperboloidal projection," Proc. Int. Conf. on Intelligent Robots and Systems, Vol.2, pp.1029-1034, 1993.

[10] H. Iwata, "Walking about virtual environments on an infinite floor," Proc. IEEE Virtual Reality, pp.286-293, 1999.

[11]. K. Kobayashi, A. Ogawa, S. L. Casner, and C. Bormann, "RTP payload format for DV (IEC 61834) video," Internet Requests For Comments, RFC 3189, 2002.

[12]. Y. Onoe, K. Yamazawa, H. Takemura, and N. Yokoya, "Telepresence by real-time view-dependent image generation from omnidirectional video streams," Computer Vision and Image Understanding, Vol.71, No.2, pp.154-165, 1998. 